\title{
Reinforced evidence on partial compatibility between Pinus sylvestris and Pinus mugo and on maternal inheritance of chloroplast DNA in the Pinus mugo $\times$ Pinus sylvestris cross
}

\author{
Andrej Kormutak ${ }^{1 *}$, Martin Galgoci ${ }^{1}$, Peter Bolecek², Dusan Gőmőry³ ${ }^{3}$ Jana Libantova ${ }^{1}$ \\ ${ }^{1}$ Plant Science and Biodiversity Center, Slovak Academy of Sciences, Institute of Plant Genetics and Biotechnology, \\ Akademicka 2, P. O. Box 39A, SK-950 07 Nitra, Slovak Republic \\ ${ }^{2}$ Constantine Philosopher University, Faculty of Natural Sciences, A. Hlinku 1, SK-949 74 Nitra, Slovak Republic \\ ${ }^{3}$ Technical University in Zvolen, Faculty of Forestry, T. G. Masaryka 24, SK-960 53 Zvolen, Slovak Republic \\ *Corresponding author: Andrej Kormutak, e-mail: nrgrkorm@savba.sk
}

\begin{abstract}
The crossability relationship between Scots pine (Pinus sylvestris L.) and mountain dwarf pine (Pinus mugo Turra) was tested under field conditions using an artificial pollination approach. There was partial compatibility between the parental species, as evidenced by the amount of filled seeds in their reciprocal crossings and in control variants from self-pollination, controlled intraspecific outcrossing and open pollination of mother trees. The crossability degree in $P$. sylvestris $\times P$. mugo was characterised by the index 0.15 , and the reciprocal crossing by the index 0.18 . Crossability of $P$. sylvestris and $P$. mugo with their putative hybrid individuals was much higher; the number of filled seeds was comparable with that of the control variants. The reciprocal crossings of $P$. sylvestris and $P$. mugo species were highlighted by the opposite inheritance of their chloroplast DNA (cpDNA). The paternal cpDNA inheritance in P. sylvestris $\times$ $P$. mugo and maternal cpDNA inheritance in $P$. mugo $\times$ P. sylvestris was repeatedly confirmed using the cpDNA trnV-trnH/Hinfl marker as well as the newly developed partial cpDNA trnV-

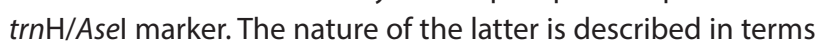
of the nucleotide sequence.
\end{abstract}

Keywords: Pinus sylvestris, Pinus mugo, hybridisation, crossability, $c p D N A$

\section{Introduction}

Extensive experiments with pine artificial hybridisation performed during the second half of the twentieth century led to the postulation of an asystematic pattern of species hybridisation within the genus Pinus (Mirov, 1967). Although taxonomically distant, some species reportedly rarely intercross, whereas species of the same taxonomic rank exhibit a variable degree of reproductive isolation. Myriad studies (Righter and Duffield, 1951; Critchfield, 1963, 1975; Saylor and Koenig, 1967) discuss intersectional pine hybrids along with a number of artificially obtained intrasectional hybrids. In this context, the crossability relationship between Scots pine ( $P$. sylvestris L.) and mountain dwarf pine (P. mugo Turra) of the section Pinus, subsection Sylvestres (Little and Critchfield, 1969) is of special interest. It directly impacts the gene flow intensity within the putative hybrid swarms of these species that occur in central and southern Europe (Prus-Głowacki and Szweykowski, 1980; Christensen, 1987). In the past, Dengler (1932, 1939), Schmidt (1951), Marcet (1967) and Moulalis et al. (1976) postulated the compatible nature of Scots and mountain dwarf pines for artificial crossings. Recently, the concept of unilateral incompatibility was inferred instead based on the gene flow from $P$. mugo to $P$. sylvestris but not in the reciprocal direction (Wachowiak et al., 2006). However, the validity of this concept is questioned by the results of mutual hybridisation of these species. The use of 
two maternal $P$. sylvestris trees in crossings with $P$. mugo resulted in a $10 \%$ yield of filled seeds; the same was true of the reciprocal $P$. mugo $\times P$. sylvestris crossing, with double the amount of filled seeds when three $P$. mugo mother trees were used. We consider these data as evidence that supports partial compatibility between $P$. sylvestris and $P$. mugo (Kormutak et al., 2017). One mother tree of each in the $P$. sylvestris $\times P$. mugo and reciprocal crossings only produces additional empty seeds, data that emphasise the role of proper parental tree selection for hybridisation.

Hybrid validation based on the trnV-trnH/Hinfl restriction profiles of the chloroplast DNA (cpDNA) revealed bidirectional cpDNA inheritance. In the $P$. sylvestris $\times P$. mugo cross, there is a commonly acknowledged paternal transmission of cpDNA into the embryos, but the reciprocal cross exhibits the maternal mode of cpDNA inheritance (Kormutak et al., 2017). There are complicated hybridological relationships between $P$. sylvestris and $P$. mugo ssp. mugo, Pinus uncinata Ramond and Pinus uliginosa Neumann of the P. mugo complex (Christensen, 1987; Wachowiak et al., 2005a), with a special reference to the controversial results of $P$. sylvestris and $P$. mugo hybridisation. Thus, the challenge made by Lewandowski and Wiśniewska (2006) is justified; they emphasise the necessity to repeat the artificial crossing experiments for several years to exclude the influence of biotic and abiotic factors on the process. In continuation of a 2013 experiment (Kormutak et al., 2017), we made additional attempts to intercross $P$. mugo with $P$. sylvestris in two successive years to strengthen the evidence on partial compatibility of their reciprocal crossings. Additionally, molecular proof for cpDNA maternal inheritance in the $P$. mugo $\times P$. sylvestris crossing based initially on the polymerase chain reaction-restriction fragment length polymorphism (PCR-RFLP) of trnV-trnH segment was extended to the nucleotide level of the respective segment.

\section{Materials and Methods}

\section{Artificial hybridisation}

The two artificial crossing experiments with Scots pine (P. sylvestris $L$.) and mountain dwarf pine ( $P$. mugo Turra) were conducted separately in 2016 and 2017. Six P. mugo trees from the natural stand of the species in the Mala Fatra mountain range (northern Slovakia) were used in the experiment (no. 6, 7, 8, 11, 12,13 ), together with two taxonomically verified $P$. mugo individuals (no. 9 and 10) from the Botanical Garden of the Technical University in Zvolen (central Slovakia). One Scots pine (no. 1) from a natural stand in Oravice (northern Slovakia) served as a mother tree in 2016, and the six additional Scots pines from natural stands in southern and western Slovakia served as pollen trees (no. 2, 3, 4, 5, 14, 15). Except for the P. mugo and P. sylvestris trees, two individuals of the putative hybrid swarm of these species $(\mathrm{H} 1, \mathrm{H} 2)$ in Zuberec (northern Slovakia) were used as pollen parents in 2016. The experiments were performed on May 5-15, 2016 and June 14-25, 2017, depending on the altitude of the localities with the respective mother trees.
Controlled pollination primarily focused on the reciprocal crossings between $P$. mugo and $P$. sylvestris, but intraspecific crossing variants were also taken into account. These variants involved self-pollination of mother trees, mutual hybridisation of different individuals of the same species and open pollination of mother trees. Freshly collected pollen from all the trees associated with hybridisation was subjected to an in vitro germination test prior to pollination to avoid using poor-quality pollen. Each sample was tested in triplicate on $1.5 \%$ agar medium with $10 \%$ sucrose (Brewbaker and Kwack, 1963). The samples were cultivated in covered Petri dishes at $25^{\circ} \mathrm{C}$ for $48 \mathrm{~h}$. Macrostrobili of the mother trees were isolated 3-4 days before they reached receptivity using paper bags with strengthened walls. Special care was taken to install the isolating bags before the initiation of pollen shedding in the respective mother and neighbour trees. Pollination of the receptive macrostrobili was performed under strictly controlled conditions using a softhair painting brush. Isolation bags were removed from pollinated macrostrobili after complete closure of their ovuliferous scales. At this stage of the experiment, an attempt to count pollinated macrostrobili in crossing variants was made. Mature cones of the mountain dwarf pine were collected in October and those of Scots pine in December of the next year following pollination. Hand extracted and dewinged seeds of individual crossing variants were counted and processed with regards to the proportions of filled and empty seeds. This was done in samples of germinating seeds which were used for DNA extraction from excised embryos. Crossability between mountain dwarf and Scots pines was expressed quantitatively as a proportion of sound seed percentage from crosses between species and a percentage of sound seed yield from within-species crosses (Critchfield, 1975).

Experimental data on pollen viability in the parental trees and seed quality in the tested variants of crossings were statistically examined using analysis of variance (ANOVA) with the procedure GLM 9.1 (SAS 2004) employed. As the crossing design was not fully factorial (father trees differed among mothers), a nested ANOVA model was used, while both mothers and parental combinations within mothers were considered fixed-effect factors. Prior to analysis, normality of residuals was tested using the Shapiro-Wilk test, while the variance was tested using the Bartlett's test. Frequency data (proportions of filled seeds, pollen germinability) were arcsinetransformed prior to analysis.

\section{DNA extraction}

Total parental tree DNA in the P. sylvestris $\times P$. mugo and reciprocal crossings was extracted from young needles using the CTAB protocol by Murray and Thompson (1980). A slightly modified protocol from that mentioned above was also used to extract DNA from hybrid embryos. Embryo DNA extraction was preceded by the in vitro seed germination in Petri dishes with wet cotton-wool paper at $25^{\circ} \mathrm{C}$ and subsequent excision of protruding embryos from surrounding megagametophyte tissue. Individual embryos were homogenised separately in 1.5 $\mathrm{ml}$ Eppendorf tubes with $250 \mu \mathrm{l}$ of extraction buffer. A small amount of extracted DNA was electrophoretically run on a 1.5 
$\%$ agarose gel in $1 \times$ Tris-Borate-EDTA (TBE) buffer, $\mathrm{pH} 8.0$, to estimate DNA concentration.

\section{$P C R$ amplification of $c P D N A$ trnV-trnH region}

A partial segment of the trnV-trnH region of cpDNA (approximately 450 base pairs [bp]) in P. sylvestris and P. mugo was amplified using the primer pair trnV-trnH4FOR (5'-TCTCGAGATAGATCTCGATCT-3') (newly designed primer) and trnV-trnH REV (5'-CTTGGTCCACTTGGCTACGT-3' (Parducci and Szmidt, 1999). A total number of the excised embryos which were subjected to analysis with this primer pair equalled 10 embryos in $P$. sylvestris $\times P$. mugo crossing and 34 embryos of $P$. mugo $\times$ P. sylvestris crossing. The $25-\mu \mathrm{l} P C R$ mixture contained $200-300 \mathrm{ng}$ plant genomic DNA, $1 \times$ PCR buffer with $25 \mathrm{mM}$ content of $\mathrm{MgCl}_{2}, 0.2 \mathrm{mM}$ deoxynucleotides (dNTPs), 10 pmol each primer and 1 U FIRE Pol DNA polymerase (Solis Biodyne, Estonia). PCR amplification was performed at $94^{\circ} \mathrm{C}$ for $3 \mathrm{~min}, 35$ cycles at $94^{\circ} \mathrm{C}$ for $30 \mathrm{~s}, 52^{\circ} \mathrm{C}$ for $30 \mathrm{~s}$ and $72^{\circ} \mathrm{C}$ for $50 \mathrm{~s}$, and a final elongation step at $72^{\circ} \mathrm{C}$ for $10 \mathrm{~min}$. To confirm successful amplification of the respective cpDNA segment, $5 \mu$ of the PCR product from each sample was separated electrophoretically in a $1 \%$ agarose gel. Fifteen $\mu \mathrm{l}$ of PCR product was digested with the restriction enzyme Asel and separated in a $2 \%$ agarose gel. Concurrently, $2 \mu \mathrm{l}$ of each $P$. mugo and $P$. sylvestris PCR product was cloned into a pJet 1.2 vector (Thermo Fisher Scientific, USA) and transformed into E. coli DH5a competent cells. Two recombinant plasmids with a respective PCR product were sequenced commercially.

Except for cpDNA validation given above, a simultaneous analysis of a cpDNA region of approximately 2.5 kilobases (kb) amplified with trnV-trnH FOR (5'-GCTCAGCAAGGTAGAGCACC-3') and trnV-trnH REV (5'-CTTGGTCCACTTGGCTACGT-3') (Parducci and Szmidt, 1999) was repeated for 15 embryos of $P$. mugo $\times P$. sylvestris and 21 embryos of the reciprocal crossing using trnV-trnH/Hinfl restriction profiles. These profiles are diagnostic for the parental species (Kormutak et al., 2017).

\section{Results}

\section{Pollen viability}

Pollen quality varied considerably among the trees involved in the 2016 hybridisation. In the four P. sylvestris individuals (no. 2-5), the pollen germination percentage was between $81.0 \%$ and $96.6 \%$. On the contrary, in P. sylvestris no. 1 it averaged only $62.6 \%$. The germination potential of $P$. mugo individuals no. 6-10 was comparable to the $P$. sylvestris individuals; they contained $73.6-94.3 \%$ of germinating pollen. Pollen tube length, a second pollen viability parameter, varied among individual trees, with a range of $75.5 \mu \mathrm{m}$ to $146.7 \mu \mathrm{m}$. There was no correlation between pollen germination percentage and pollen tube length in individual trees. For example, P. sylvestris no. 1, with $62.6 \%$ average germination of its pollen, possessed pollen tubes with a maximal length of $146.7 \mu \mathrm{m}$, whereas the pollen of $P$. sylvestris no. 2, with $96.6 \%$ germination, reached only
$78.3 \mu \mathrm{m}$ average pollen tube length. Putative hybrid individuals $\mathrm{H} 1$ and $\mathrm{H} 2$ were exceptions in this respect. The average germination of their pollen was $53.6 \%$ and $27.6 \%$, and the corresponding lengths of their pollen tubes were $71.7 \mu \mathrm{m}$ and 27.6 $\mu \mathrm{m}$, respectively; they deviated conspicuously from the rest of trees in 2016. ANOVA confirmed statistical significance of the differences in pollen germination percentage among $P$. sylvestris, P. mugo and putative hybrid individuals $(P=0.0005)$ as well as among $P$. sylvestris and $P$. mugo individuals $(P<0.0001)$. Similarly, the variation in pollen tube length among the paternal trees was statistically significant $(P<0.0001)$. Artificial pollination in 2017 involved three P. mugo (no. 11-13) and two P. sylvestris (no. 14 and 15) individuals with comparable pollen quality. Germination percentage was 91.6-97.3\%, and the corresponding pollen tube length was 87.0-96.5 $\mu \mathrm{m}$. Altogether, 12 parental trees were involved in the pollen quality estimation in 2016 and five in 2017. The pollen with outlined viability was subsequently used in artificial pollination experiments in the respective years.

\section{Crossability}

The results of crossing experiments performed in 2016 are summarised in Table 1 and those obtained in 2017 in Table 2. Efficiency assessment of the reciprocal crossings between Scots and mountain dwarf pines is based on one maternal and paternal tree in $P$. sylvestris $1 \times P$. mugo 9 crossing and on six mother mountain dwarf pines and six paternal Scots pines used in the $P$. mugo $\times P$. sylvestris crossings. Using seed quality as the main criterion of hybridisation affinity between species and trees involved in hybridisation, a low degree of compatibility could be postulated for the $P$. sylvestris $1 \times P$. mugo 9 crossing. The crossing yielded $15.5 \%$ of filled seeds and an average of 1.7 seeds per cone. The corresponding characteristics in a controlled outcrossing of $P$. sylvestris $1 \times P$. sylvestris 2 averaged $98.0 \%$ of filled seeds and 18.7 seeds per cone. In quantitative terms, the crossability of $P$. sylvestris $\times P$. mugo combination was characterised by the index 0.158 .

A highly variable crossability pattern was characteristic for the reciprocal $P$. mugo $\times P$. sylvestris crossings; the paternal trees exerted a profound effect on the crossability. The amount of fully developed seeds obtained within six $P$. mugo mother trees (no. 6, 7, 8, 11, 12, 13) that were pollinated with P. sylvestris pollen varied widely. Of the 10 attempted $P$. mugo $\times P$. sylvestris crossings, there were no filled seeds obtained from 30 mature cones of the four variants (P. mugo $6 \times P$. sylvestris 3, P. mugo $8 \times$ P. sylvestris 4, P. mugo $12 \times P$. sylvestris 15 and P. mugo $13 \times P$. sylvestris 14 ). For $P$. mugo $6 \times P$. sylvestris 5 , only one mature cone was collected that contained one filled seed. Likewise, in $P$. mugo $7 \times P$. sylvestris 2 , with two mature cones harvested, there were three seeds of which two seeds were fully developed. The remaining four variants were remarkable for the increased number of collected mature cones, the total number of obtained seeds and percentage of fully developed seeds (Tables 1 and 2). P. mugo $8 \times P$. sylvestris 3 produced $2.2 \%$ of filled seeds, $P$. mugo $11 \times P$. sylvestris 15 yielded $9 \%$ of filled seeds and $P$. mugo $13 \times P$. sylvestris 15 produced a higher amount of filled seeds (50.6\%). However, P. mugo $7 \times P$. sylvestris 5 was the most 
efficient cross, with $85.2 \%$ of filled seeds. The crossability indexes calculated for the crossing variants $P$. mugo $8 \times P$. sylvestris 3, P. mugo $13 \times P$. sylvestris 15 and $P$. mugo $7 \times P$. sylvestris 5 and P. mugo $11 \times P$. sylvestris 15 were $0.25,0.63,0.90$ and 0.11 , respectively, and averaged 0.38 . This crossability degree was twice as high as that calculated for the $P$. sylvestris $1 \times P$. mugo 9 crossing.

The obtained experimental data proved unequivocally that crossability variation between mountain dwarf and Scots pines is due to differentiated hybridisation affinity of the individual pairs of parental trees used in hybridisation. The most expressive examples of the kind are the crossings of $P$. mugo 7 mother tree with the paternal trees $P$. sylvestris 2 and $P$. sylvestris 5 , which had comparable pollen viability. Crossing with $P$. sylvestris 2 failed completely, whereas the crossing of $P$. mugo 7 with $P$. sylvestris 5 generated the highest percentage of filled seeds (Table 1). A similar situation was also observed for the mother tree $P$. mugo 13, which in crossings with $P$. sylvestris 14 and $P$. sylvestris 15 produced empty seeds and $50.6 \%$ of filled seeds, respectively. For paternal tree $P$. sylvestris 15 , crossings with the maternal trees $P$. mugo 11 and $P$. mugo 12 produced 9 and $0 \%$ of filled seeds. This data supports the concept of differential hybridisation affinity between the parental trees. This option also satisfies the crossings of Scots and mountain dwarf pine mother trees with the putative hybrid individuals $\mathrm{H} 1$ and $\mathrm{H} 2$ (which were conducted in 2016). Both pollen trees exhibited lowered pollen viability ( $53.6 \%$ and $27.6 \%$, respectively). Nevertheless, the crossing of $\mathrm{H} 1$ pollen tree with mother trees P. sylvestris 1 and P. mugo 7 resulted in $87.7 \%$ and $66.6 \%$ of fully developed seeds. The use of pollen tree $\mathrm{H} 2$ in a crossing with $P$. mugo 6 yielded $92.5 \%$ of filled seeds, but it failed to produce seeds when crossed with P. mugo 8 mother tree (Table 1). ANOVA revealed that differences in the filled seed percentage of the crossing variants attempted in individual mother trees were statistically significant $(P<0.0001)$.

At the macrostrobili level, there is a marginally significant relationship between crossability and pollinated macrostrobili survival level $(r=0.509 ; P=0.063)$ across parental combinations. According to Sarvas (1962), a limiting factor for pollinated macrostrobili survival during winter is the number of ovules in a given macrostrobilus with pollen tubes that penetrate the nucellar tissue of an ovule. This factor provides physiological stimuli for further ovule development during the next spring. When pollen grains are unable to germinate on the nucellus due to an incompatible reaction between them, ovule desiccation and dropping follow soon after pollination (Kormutak, 1984). In the present study, increased macrostrobili dropping was registered in the reciprocal crossings of $P$. sylvestris and $P$. mugo compared to the corresponding control variants. This observation was particularly true for the crossings attempted in mother $P$. sylvestris $1, P$. mugo 6, P. mugo 7 and $P$. mugo 13, with 20.4-96.4\% dropping (Tables 1 and 2). The only exceptions were the P. mugo $8 \times P$. sylvestris 3 and P. mugo $8 \times P$. sylvestris 4 crossings, which were accompanied by reduced macrostrobili dropping (41.9\% and $51.6 \%$ ) compared to the outcrossed control (64.8\%). In spite of an increased rate of macrostrobili survival, the first of the crossings mentioned above produced only $2.2 \%$ of filled seeds whereas the second crossing was a complete failure.

Summarily, the efficiency of artificial crossing experiments conducted during 2016-2017 with P. mugo mother trees is given in Table 3. P. mugo $\times$ P. sylvestris crossings shared the lowest crossability characteristics, as evidenced by the lowest percentage of filled seeds and the highest dropped macrostrobili. The reverse observations were characteristic of the control crossing variant $P$. mugo $\times P$. mugo, with a greater amount of filled seeds and less dropped macrostrobili. The $P$. mugo with $\mathrm{H} 1$ and $\mathrm{H} 2$ pollen trees occupied an intermediate position between the above situations. Based on the experimental data, we may conclude that partial compatibility of the reciprocal crossings between $P$. sylvestris and $P$. mugo is an essential feature of their reproductive behaviour.

\section{Hybrid validation}

Reciprocal $P$. sylvestris and $P$. mugo crossings exhibit bimodal cpDNA inheritance, as evidenced by the paternal cpDNA transmission in $P$. sylvestris $\times P$. mugo crossings and maternal inheritance in the reciprocal crossing (Kormutak et al., 2017). The evidence is based on the trnVtrn $\mathrm{H} /$ Hinfl restriction profiles of the corresponding hybrids and their parents. The PCR fragments of 2,539 and 2,544 bp, characteristic of $P$. mugo and $P$. sylvestris, respectively, were amplified using trnV-trnH primers. They cover the region between the $\operatorname{trnV}$ (UAC) and $t r n \mathrm{H}$ (GUG) genes and involve six open reading frames (orf64a, orf56b, orf66, orf67a, orf133c and orf57c) and several hundred bp of noncoding cpDNA (Parducci and Szmidt, 1999). Digestion of corresponding PCR products with restriction enzyme Hinfl generates restriction DNA profiles that differ in $P$. sylvestris and $P$. mugo (Kormutak et al., 2002). Based on DNA sequence data in the NCBI GeneBank database for $P$. sylvestris (KR476379.1) and $P$. mugo (JN854181.1), as many as 13 fragments are expected to be generated that range from 8 to $714 \mathrm{bp}$. The diagnostic fragments for $P$. sylvestris, $P$. mugo and their hybrids were 275, 276 and 282 bp, respectively, as revealed in $8 \%$ polyacrylamide gels (Figs. 1- 2 ).

We further elucidated maternal cpDNA inheritance in $P$. mugo $\times P$. sylvestris crossings. We designed a new primer pair that covers only the most variable (partial) cpDNA region with a considerably shortened PCR product (approximately $0.5 \mathrm{~kb}$ ). Restriction analysis of the PCR product with Asel enabled discrimination between $P$. sylvestris and $P$. mugo. The latter possesses an Asel-specific restriction site that is missing in the amplified $P$. sylvestris DNA sequence. As a result, the Asel digest produces 202-bp and 273-bp fragments characteristic of $P$. mugo and an intact 481-bp fragment characteristic of $P$. sylvestris. With a $2 \%$ agarose gel, we illustrated paternal cpDNA inheritance in $P$. sylvestris $\times P$. mugo crossing (Fig. $3 \mathrm{~A}$ ) and maternal cpDNA inheritance in $P$. mugo $\times P$. sylvestris crossing (Fig. 3B) with identical restriction profiles in the paternal tree $P$. mugo and hybrid embryo and in the maternal tree $P$. mugo and hybrid embryo. 
Table 1

Results of artificial pollination of P. sylvestris and P. mugo mother trees in 2016

\begin{tabular}{|c|c|c|c|c|c|c|}
\hline Crossing variants & $\begin{array}{c}\text { Number of pollinated } \\
\text { macrostrobili }\end{array}$ & $\begin{array}{l}\text { Number of collected } \\
\text { mature cones }\end{array}$ & $\begin{array}{l}\text { Percent of macro- } \\
\text { strobili dropping }\end{array}$ & $\begin{array}{l}\text { Total number of } \\
\text { obtained seeds }\end{array}$ & $\begin{array}{l}\text { Number of seeds } \\
\text { per cone }\end{array}$ & $\begin{array}{l}\text { Percent of } \\
\text { filled seeds }\end{array}$ \\
\hline P. sylvestris 1 - selfing & 29 & 23 & 20.7 & 70 & 3.0 & 9.09 \\
\hline P. sylvestris $1 \times P$. sylvestris 2 & 35 & 29 & 17.2 & 543 & 18.7 & 98.0 \\
\hline P. sylvestris $1 \times$ P. mugo 9 & 118 & 94 & 20.4 & 161 & 1.7 & 15.5 \\
\hline P. sylvestris $1 \times \mathrm{H} 1$ & 56 & 44 & 21.5 & 650 & 14.7 & 87.7 \\
\hline P. sylvestris 1 - open pollination & - & 26 & - & 141 & 5.4 & 42.0 \\
\hline P. mugo 6 - selfing & 30 & 25 & 16.7 & 926 & 37.0 & 22.0 \\
\hline P. mugo $6 \times$ P. mugo 9 & 30 & 22 & 26.7 & 625 & 28.4 & 86.9 \\
\hline P. mugo $6 \times$ P. sylvestris 5 & 28 & 1 & 96.4 & 1 & 1.0 & 0 \\
\hline P. mugo $6 \times$ P. sylvestris 3 & 35 & 6 & 82.9 & 2 & 0.3 & 0 \\
\hline P. mugo $6 \times \mathrm{H} 2$ & 45 & 34 & 24.5 & 747 & 21.9 & 92.5 \\
\hline P. mugo 6 - open pollination & - & 31 & - & 1200 & 38.7 & 78.0 \\
\hline P. mugo 7 - selfing & 12 & 7 & 41.7 & 83 & 11.8 & 87.7 \\
\hline P. mugo $7 \times$ P. mugo 6 & 17 & 15 & 11.8 & 247 & 16.4 & 94.3 \\
\hline P. mugo $7 \times$ P. sylvestris 2 & 32 & 2 & 93.7 & 3 & 1.5 & 0 \\
\hline P. mugo $7 \times P$. sylvestris 5 & 23 & 6 & 74.0 & 115 & 19.1 & 85.2 \\
\hline P. mugo $7 \times \mathrm{H} 1$ & 38 & 11 & 71.1 & 75 & 6.8 & 66.6 \\
\hline P. mugo 7 - open pollination & - & 21 & - & 794 & 37.8 & 76.0 \\
\hline P. mugo 8 - selfing & 53 & 35 & 34.0 & 193 & 5.5 & 38.4 \\
\hline P. mugo $8 \times$ P. mugo 10 & 34 & 12 & 64.8 & 34 & 2.8 & 8.8 \\
\hline P. mugo $8 \times$ P. sylvestris 3 & 55 & 32 & 41.9 & 106 & 3.3 & 2.2 \\
\hline P. mugo $8 \times$ P. sylvestris 4 & 33 & 16 & 51.6 & 28 & 1.7 & 0 \\
\hline P. mugo $8 \times \mathrm{H} 2$ & 52 & 10 & 80.8 & 6 & 0.6 & 0 \\
\hline P. mugo 8 - open pollination & - & 32 & - & 420 & 13.1 & 94.0 \\
\hline
\end{tabular}

Table 2

Results of artificial pollination of P. mugo mother trees in 2017

\begin{tabular}{|c|c|c|c|c|c|c|}
\hline Crossing variants & $\begin{array}{c}\text { Number of pollinated } \\
\text { macrostrobili }\end{array}$ & $\begin{array}{l}\text { Number of collected } \\
\text { mature cones }\end{array}$ & $\begin{array}{l}\text { Percent of macro- } \\
\text { strobili dropping }\end{array}$ & $\begin{array}{l}\text { Total number of } \\
\text { obtained seeds }\end{array}$ & $\begin{array}{l}\text { Number of seeds } \\
\text { per cone }\end{array}$ & $\begin{array}{l}\text { Percent of } \\
\text { filled seeds }\end{array}$ \\
\hline P. mugo 11 - selfing & 26 & 5 & 80.7 & 190 & 38.0 & 78.3 \\
\hline P. mugo $11 \times$ P. sylvestris 15 & 32 & 20 & 37.5 & 258 & 12.9 & 9.0 \\
\hline P. mugo 11 - open pollination & - & 28 & - & 936 & 33.4 & 88.7 \\
\hline P. mugo 12 - selfing & 23 & 3 & 86.9 & 80 & 26.6 & 94.4 \\
\hline P. mugo $12 \times$ P. sylvestris 15 & 48 & 2 & 95.8 & 29 & 14.5 & 0 \\
\hline P. mugo 12 - open pollination & - & 12 & - & 631 & 52.6 & 96.2 \\
\hline P. mugo $13 \times$ P. mugo 12 & 19 & 15 & 21.0 & 659 & 43.9 & 80.1 \\
\hline P. mugo $13 \times$ P. sylvestris 14 & 14 & 6 & 57.1 & 129 & 21.5 & 0 \\
\hline P. mugo $13 \times$ P. sylvestris 15 & 32 & 19 & 40.6 & 518 & 27.2 & 50.6 \\
\hline P. mugo 13 - open pollination & - & 16 & - & 604 & 37.7 & 96.8 \\
\hline
\end{tabular}

Table 3

Pooled data on artificial pollination of P. mugo mother trees in 2016-2017

\begin{tabular}{|c|c|c|c|c|c|c|}
\hline Crossing variants & $\begin{array}{c}\text { Number of pollinated } \\
\text { macrostrobili }\end{array}$ & $\begin{array}{l}\text { Number of collected } \\
\text { mature cones }\end{array}$ & $\begin{array}{l}\text { Percent of macro- } \\
\text { strobili dropping }\end{array}$ & $\begin{array}{c}\text { Total number of } \\
\text { obtained seeds }\end{array}$ & $\begin{array}{c}\text { Number of seeds } \\
\text { per cone }\end{array}$ & $\begin{array}{l}\text { Percent of } \\
\text { filled seeds }\end{array}$ \\
\hline P. mugo - selfing & 144 & 75 & 48.0 & 1472 & 23.7 & 64.1 \\
\hline P. mugo $\times$ P. mugo & 100 & 64 & 36.0 & 1565 & 22.8 & 67.5 \\
\hline P. mugo $\times$ P. sylvestris & 332 & 110 & 66.9 & 1189 & 10.3 & 14.7 \\
\hline P. mugo $\times \mathrm{H}$ & 135 & 55 & 59.2 & 828 & 9.7 & 53.0 \\
\hline P. mugo - open pollination & - & 140 & - & 4585 & 35.5 & 88.4 \\
\hline
\end{tabular}




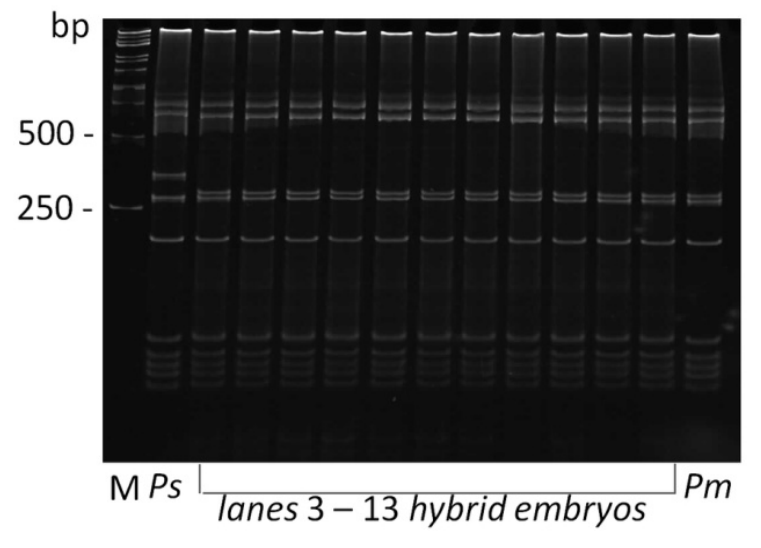

Fig. 1

Restriction profiles of cpDNA segment trnV-trnH/Hin$f$ in hybrid embryos $P$. sylvestris $\times P$. mugo. Lane Ps $-P$. sylvestris mother tree, lanes 3-13 - hybrid embryos and lane 14 - P. mugo pollen tree; $\mathrm{M}$ - size marker

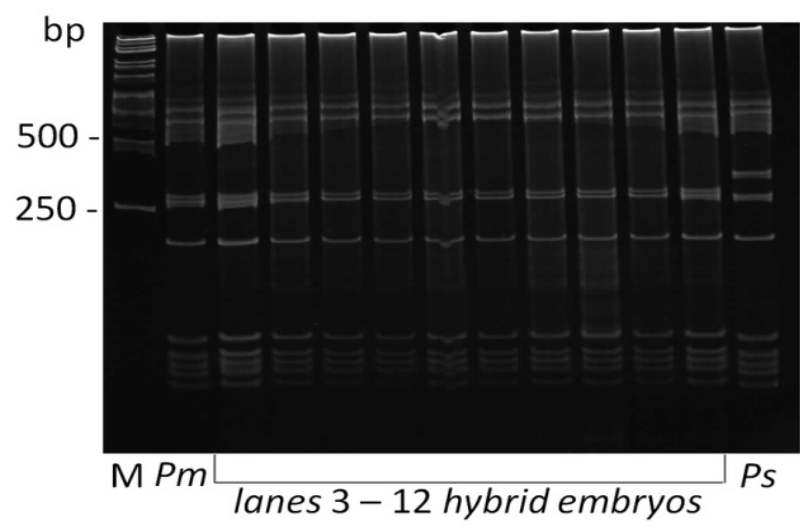

Fig. 2

Restriction profiles of cpDNA segment trnV-trnH/Hinfl in hybrid embryos $P$. mugo $\times$ P. sylvestris. Lane Pm $-P$. mugo mother tree, lanes 3-12 - hybrid embryos and lane 13 $P$. sylvestris pollen tree; $M$ - size marker
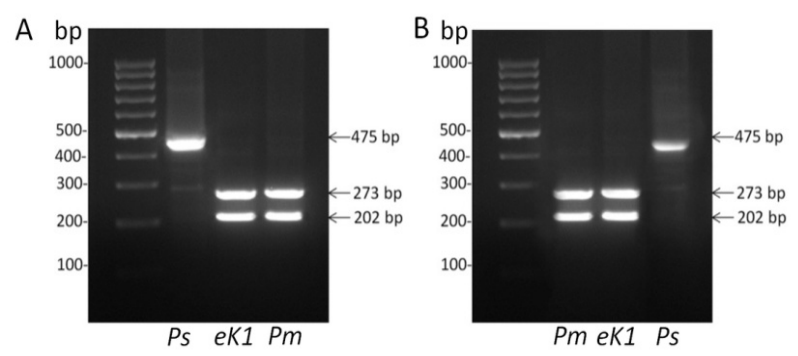

Fig. 3

Restriction profiles of partial cpDNA segment trnVtrnH4 FOR - trnV-trnH REV/Asel in P. sylvestris mother tree (PS), hybrid embryo (eK1) and P. mugo pollen tree $(P m)(A)$ and in $P$. mugo mother tree $(P m)$, hybrid embryo (eK1) and $P$. sylvestris pollen tree $(P s)(B)$; The marker is Gene Ruler 100 bp DNA Ladder (Thermo Fisher Scientific)
Concurrently, the PCR products $\mathrm{K} 1, \mathrm{~K} 2$ and $\mathrm{K} 3$ that corresponded to $P$. mugo, P. sylvestris and hybrid embryos were cloned into pJet 1.2 vector and used to transform Escherichia coli, followed by plasmid isolation and sequencing. As shown in Fig. S1, the DNA sequence of hybrid $P$. mugo $\times P$. sylvestris embryos corresponded to both the $P$. mugo mother tree DNA and the $P$. mugo DNA respective region (JN854181.1) deposited in NCBI GenBank.

\section{Discussion}

In his crossing attempt, Christensen (1987) examined the patterns of local and geographic variation in the P. mugo complex and $P$. sylvestris to determine their relationships and the extent to which hybridisation occurs between them. We extended this approach in a more straightforward way to determine the genetic relationship between $P$. mugo and $P$. sylvestris using artificial hybridisation experiments to estimate the degree of their hybridisation affinity. The obtained hybridological data confirm the claims made by some authors that the frequency of hybridisation and introgression in mixed $P$. mugo and $P$. sylvestris stands is apparently low (Christensen, 1987; Jasińska et al., 2010). In an overwhelming majority of the crossings attempted in this study, the crossability parameters of the reciprocal $P$. mugo and $P$. sylvestris crossings were conspicuously lower compared with control. At the macrostrobili level, the gametophytic incompatibility that operates as the principal reproductive barrier between the parental species (Kormutak, 1984) precluded many macrostrobili from their normal development. Due to an insufficient number of properly pollinated eggs, such macrostrobili desiccate and drop at the end of the first vegetation period (Sarvas, 1962). Surviving macrostrobili resume their growth during the next spring; this phenomenon represents the first symptom of partial compatibility between $P$. mugo and $P$. sylvestris. In P. sylvestris $\times P$. mugo crossings, the percentage of macrostrobili dropping was slightly increased compared with the controlled outcrossing of $P$. sylvestris. In the reciprocal crossings, this figure was doubled relative to control. However, the most important evidence that supports partial compatibility between mountain dwarf and Scots pines is seed quality in their reciprocal crossings, which amounted to $15.5 \%$ of filled seeds in P. sylvestris $\times$ P. mugo and $14.7 \%$ seeds of the same quality in the reciprocal crossings. Comparable results were also reported by Moulalis et al. (1976) in their attempted $P$. sylvestris $\times P$. mugo crossing. On the contrary, Wachowiak et al. (2005a) obtained only $0.9 \%$ of filled seeds from a total of 440 seeds with the same crossing. In the reciprocal $P$. mugo $\times P$. sylvestris crossing, the authors refer to three filled seeds that developed from 50 pollinated macrostrobili in the two mother trees.

Irrespective of a low or high amount of filled seeds in the reciprocal $P$. mugo and $P$. sylvestris crossings, the presented data substantiate the existence of partial compatibility between these species. According to Heiser (1949), introgressive 
hybridisation is reciprocal, but the primary genetic infiltration seems to be into $P$. sylvestris. Our data corroborate this supposition, as evidenced by a relatively high share of filled seeds in $P$. sylvestris $\times P$. mugo. In the reciprocal $P$. mugo $\times P$. sylvestris crossing, this share was comparable based on six $P$. mugo mothers and 10 attempted $P$. mugo $\times P$. sylvestris crossings. Seven of these attempts yielded only negligible amounts of filled seeds whereas the remaining three attempts produced substantionally higher proportions of filled seeds. These findings indicate that incompatibility between $P$. mugo and $P$. sylvestris is not shared equally by the individual trees of the species. Rather, the trees are differentiated in this respect and exhibit different hybridisation affinity when intercrossed.

Within the context of complicated hybridological relationships between taxa of the P. mugo complex and P. sylvestris, there is some dispute about the relative hybrid swarm hypothesis. In previous studies, Wachowiak et al. (2006) and Wachowiak and Prus-Głowacki (2008) proved a low hybridisation frequency within the hybrid swarm and postulated only unidirectional gene flow between $P$. sylvestris and $P$. mugo, with P. mugo acting as a pollen donor. Our data do not corroborate this conclusion, as evidenced by a remarkably high yield of sound seeds in some combinations of the parental $P$. mugo $\times P$. sylvestris crossing. This finding is strengthened by a positive validation of the hybrid nature of sound seeds in which both less and more efficient combinations of the $P$. mugo $\times P$. sylvestris parental trees were involved. According to Adams et al. (1988), a reliable control of crossings is of immense importance in tree-improvement programmes to avoid invalid crosses that could severely reduce anticipated genetic gains. The hybrid seeds subjected to analysis shared uniform cpDNA restriction profiles, identical with the profile of $P$. mugo mother trees, provides convincing proof for the maternal cpDNA inheritance in the respective crossing, as previously reported by Kormutak et al. (2017, 2018). According to Owens and Morris (1990), the cytological mechanism of maternal cpDNA exclusion from zygotes in $P$. menziesii is essentially the destruction of maternal plastids during egg development. In the crossing $P$. mugo $\times P$. sylvestris this mechanism does not function. It remains to be explained. Determining the species-specific cpDNA trnV-trnH/ Hinfl marker sequencing in $P$. sylvestris and $P$. mugo resulted in the identification of a new cpDNA marker that is also diagnostic for the parental species mentioned above. The partial cpDNA trnV-trnH/Asel marker, together with those of trnVtrnH/Hinfl (Kormutak et al., 2002) and trnL-trnF/Dral (Wachowiak et al., 2005b), are currently the only known tools that are applicable in verification of the interspecific crosses between $P$. sylvestris and $P$. mugo and in following the process of their introgression.

The last-mentioned aspect of hybridisation behaviour of $P$. mugo, $P$. sylvestris and their putative hybrids was examined experimentally by pollinating $P$. sylvestris and $P$. mugo maternal trees with the pollen of two putatively hybrid individuals. The obtained hybridological data revealed a high degree of genomic homology between putative hybrids and their parents. The amounts of fully developed seeds were remarkably high in the crossings of $P$. sylvestris and $P$. mugo with putative hybrids; they approached or equalled those in the controlled intraspecific outcrossing of the maternal species. In particular it is true of the $P$. mugo $6 \times \mathrm{H} 2$ combination which was exceptional in its efficiency yielding a higher amount of sound seeds than outcrossed control P. mugo $6 \times P$. mugo 9. It is reasonable to ascribe this effect of hybridisation to a high degree of genome homology of the participating parental trees of $P$. mugo $6 \times \mathrm{H} 2$ crossing, probably due to repeated backcrossing of the $\mathrm{H} 2$ individual with P. mugo trees. However, a complete failure of the crossing $P$. mugo $8 \times \mathrm{H} 2$ does not support this option, indicating rather the differences in specific combining abilities of the parental trees. It is worth of mentioning in this connection that hybrid swarm individuals of the five-needle pines $P$. sibirica and $P$. pumila yielded in controlled crossings with the parental species significantly lower amount of filled seeds in comparison with the open pollinated progenies of the species mentioned above (Vasilyeva and Goroshkevich, 2013). The same applies also for the spontaneous hybrids of these species in the Baikal region in which even more profound differences in seed quality from open pollination of the parental species $P$. sibirica and $P$. pumila on the one hand and their hybrids on the other hand were reported by Vasilyeva et al. (2020). Also, the attempt by Lewandowski and Wiśniewska (2006) to hybridise the peatbog pine, named $P$. uliginosa, with its putative parental species P. sylvestris and P. mugo. A small-scale experiment based on nine total macrostrobili finished successfully only when combined with P. mugo. Macrostrobili of the peat-bog pine pollinated with P. sylvestris pollen aborted completely at the conelet stage. It is obvious that satisfactory elucidation of the intricate hybridisation pattern within this group of taxa requires a more complex approach with a higher number of the involved parental trees and consideration of the combining ability of each of them.

\section{Conclusions}

The presented hybridisation data proved convincingly the partial compatibility between $P$. sylvestris and $P$. mugo. The degree of their crossability varied considerably depending on the parental trees under hybridisation. Reciprocal crossings exhibited the opposite mode of cpDNA inheritance, a phenomenon that should be taken into account when following gene flow in the contact zones of the species. A remarkably high crossability of the parental species $P$. sylvestris and $P$. mugo with their putative hybrid individuals may provide indirect evidence that indicates the truly hybrid nature of the latter. We believe that the newly derived cpDNA marker, which is diagnostic for $P$. sylvestris and $P$. mugo, will facilitate monitoring of their introgression and gene flow among the introgressants.

\section{Acknowledgements}

This study was funded by the VEGA Grant Agency, project no. $2 / 0022 / 20$. A special thank to the Proof-Reading-Service in Hertfordshire, UK for proofreading the paper. 


\section{References}

Adams WT, DB Neale and CA Loopstra (1988) Verifying controlled crosses in conifer tree-improvement programs. Silvae Genetica 37(3-4): 147-152

Brewbaker JL and BH Kwack (1963) The essential role of calcium ion in pollen germination and pollen tube growth. American Journal of Botany 50(9): 859-865. https://doi.org/10.1002/j.1537-2197.1963.tb06564.x

Christensen KI (1987) Taxonomic revision of the Pinus mugo complex and P. $\times$ rhaetica (P. mugo $\times$ sylvestris) (Pinaceae). Nordic Journal of Botany 7(4): 383-408. https://doi.org/10.1111/j.1756-1051.1987.tb00958.x

Critchfield WB (1963) The Austrian x red pine hybrid. Silvae Genetica 12(6): 187 191

Critchfield WB (1975) Interspecific hybridization in Pinus: a summary review. In: Fowler DP and CY Yeatman (eds) Symposium on Interspecific and Interprovenance Hybridization in Forest Trees, Proceedings of the 14th Meeting, Canadian Tree Improvement Association, Part II, Fredericton, pp 99-105

Dengler A (1932) Künstliche Bestäubung Versuche an Kiefern. Zeitschrift für Forst- und Jagdwesen 64: 513-555

Dengler A (1939) Über die Entwicklung künstlicher Kiefernkreuzungen. Zeitschrift für Forst- und Jagdwesen 71: 457-485

Heiser CB (1949) Natural hybridization with particular reference to introgression. The Botanical Review 15: 645-687. https://doi.org/10.1007/bf02861577

Jasińska A, W Wachowiak, E Muchewicz, K Boratyńska, JM Montserrat and A Boratyński (2010) "Cryptic" hybrids between P. uncinata and P. sylvestris. Botanical Journal of the Linnean Society 163(4): 473-485. https://doi.org/10.1111/j.1095-8339.2010.01065.x

Kormutak A (1984) Some cytological and biochemical aspects of interspecific incompatibility in pines (Pinus sp.). Acta Dendrologica 7: 1-92

Kormutak A, B Vookova, T Salajova, XR Wang and A Szmidt (2002) Morphometric and genetic analyses of the putative hybrid Pinus sylvestris $\times$ Pinus mugo in Habovka. In: IUFRO Symposium on Population and Evolutionary Genetics of Forest Trees, 25-29 August 2002, Stara Lesna, Slovakia, pp. 29

Kormutak A, M Galgoci, P Manka, M Koubova, M Jopcik, D Sukenikova, P Bolecek and D Gömöry (2017) Field-based artificial crossings indicate partial compatibility of reciprocal crosses between Pinus sylvestris and Pinus mugo and unexpected chloroplast DNA inheritance. Tree Genetics and Genomes 13 68. https:// doi.org 10.1007/s11295-017-1152-x

Kormutak A, M Galgoci, D Sukenikova, P Bolecek, J Libantova and D Gömöry (2018) Maternal inheritance of chloroplast DNA in Pinus mugo Turra: a case study of Pinus mugo $\times$ Pinus sylvestris crossing. Plant Systematics and Evolution 304(1): 71-76. https://doi.org/10.1007/s00606-017-1449-0

Lewandowski A and M Wiśniewska (2006) Short note: crossability between Pinus uliginosa and its putative parental species Pinus sylvestris and Pinus mugo. Silvae Genetica 55(2): 52-54. https://doi.org/10.1515/sg-2006-0008

Little EL and WB Critchfield (1969) Subdivisions of the genus Pinus (pines). US Department of Agriculture, Forest Service, Miscellaneous Publication no. 1144, pp 1-51

Marcet E (1967) Über den Nachweis spontaner Hybriden von Pinus mugo Turra und Pinus silvestris L. aufgrund von Nadelmerkmalen. Berichte der Schweizerischen Botanischen Gesellschaft 77: 314-361

Mirov NT (1967) The genus Pinus. The Ronald Press Company, New York, 602 , OCLC Number 712344. https://doi.org/10.2307/4004229

Moulalis D, C Bassiotis and D Mitsopoulos (1976) Controlled pollinations among pine species in Greece. Silvae Genetica 25(3-4): 95-107

Murray MG and WF Thompson (1980) Rapid isolation of high molecular weight DNA. Nucleic Acid Research 8(19): 4321-4325. https://doi.org/10.1093/nar/8.19.4321

Owens JN and S J Morris (1990) Cytological basis for cytoplasmic inheritance in Pseudotsuga menziesii. I. Pollen tube and archegonial development. American Journal of Botany 77(4): 433-445. https://doi.org/10.1002/j.1537-2197.1990.tb13574.x

Parducci L and A Szmidt (1999) PCR-RFLP analysis of cpDNA in the genus Abies. Theoretical and Applied Genetics 98: 802-808. https://doi.org/10.1007/s001220051137

Prus-Głowacki W and J Szweykowski (1980) Serological characteristic of some putative hybrid individuals from a P. sylvestris $\times$ P. mugo swarm population. Acta Sociatis Botanicorum Poloniae 49(1-2): 127-142.

https://doi.org/10.5586/asbp.1980.011
Righter FI and JW Duffield (1951) Inter-species hybrids in pine. Journal of Heredity 42(2): 75-80. https://doi.org/10.1093/oxfordjournals.jhered.a106169 Sarvas R (1962) Investigations on the flowering and seed crop of Pinus sylvestris. Communicationes Instituti Forestalis Fenniae 53: 51-198

Saylor RC and RL Koenig (1967) The slash x sand pine hybrid. Silvae Genetica 16(4): 134-138

Schmidt E (1951) Die aufrechten Bergföhren in der Schweiz. Schweiz Beitr Dendrol 3: 9-13

Vasilyeva GV and SN Goroshkevich (2013) Crossability of Pinus sibirica and P. pumila with their hybrids. Silvae Genetica 62(1-2): 61-68. https://doi.org/10.1515/sg-2013-0008

Vasilyeva G, A Bondar and S Goroshkevich (2020) What does a mixed population of Pinus sibirica and P. pumila from the southern Baikal region suggest about the structure of their hybrid zone? European Journal of Forest Research139: 311-319. https://doi.org/10.1007/s10342-019-01254-7

Wachowiak W, K Celiński and W Prus-Głowacki (2005a) Evidence of natural recip rocal hybridisation between Pinus uliginosa and P. sylvestris in the sympatric population of the species. Flora 200: 563-568. https://doi.org/10.1016/j.flora.2005.06.007

Wachowiak W, A Lewandowski and W Prus-Głowacki (2005b) Reciprocal controlled crosses between Pinus sylvestris and P. mugo verified by a species-specific cpDNA marker. Journal of Applied Genetics 46(1): 41-43

Wachowiak W, J Odrzykosli, Ł Myczko and W Prus-Głowacki (2006) Lack of evidence on hybrid swarm in the sympatric population of Pinus mugo and $\mathrm{P}$. Sylvestris. Flora 201: 307-316. https://doi.org/10.1016/j.flora.2005.08.004 Wachowiak W and W Prus-Głowacki (2008) Hybridization processes in sympatric populations of pines Pinus sylvestris L., P. mugo Turra and P. uliginosa Neumann. Plant Systematics and Evolution 271: 29-40. https://doi.org/10.1007/s00606-007-0609-z 